\title{
Tumours of the chest in children: A review
}

A C Jeevarathnum, MB BCh, FCPaed (SA), Dip Allergy (SA), MMed, Cert Paed Pulm (SA), European Respiratory Diploma; A van Niekerk, MB BCh, MMed; D Parris, BSc, MB BCh, FCPaed (SA), Dip Allergy (SA); K De Campos, MB ChB, MMed, Dip Allergy (SA); W Wijnant, MD Paed, Dip Allergy (SA), Cert Paed Pulm (SA); X Deadren, MB ChB, FCPaed (SA), MMed; A Büchner, MB ChB, DCH (SA), FCPaed (SA), MMed, Dip Pall Med, Cert Med Oncol (Paed)(SA);

F Omar, MB ChB, FCPaed (SA), Cert Paed Med, Onc Paed (SA);

D Reynders, MB ChB, FCPaed (SA), MRCPCH, Cert Paed Med, Onc Paed (SA); R J Green, PhD, DSc

Department of Paediatrics and Child Health, School of Medicine, Faculty of Health Sciences, University of Pretoria and Steve Biko Academic Hospital, Pretoria, South Africa

Corresponding author: A C Jeevarathnum (acjeevarathnum@gmail.com)

S Afr Respir J 2016;22(1):7-11. DOI: 10.7196/SARJ.2016.v22i1.59

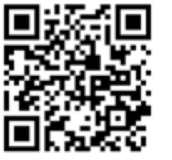

Tumours of the chest in children constitute an array of pathology and clinical symptomatology. These tumours can be benign or malignant, cystic or solid. If malignant they can be primary or as a result of secondary metastases. Collectively, tumours of the chest in children, whether benign or malignant, are very rare. The exact incidence is largely unknown globally. Primary malignant tumours as an entity of their own constitute $0.2 \%$ of malignancies in the paediatric population. ${ }^{[1]}$ Non-neoplastic lesions of the lung including bronchogenic cysts, sequestrations, congenital pulmonary airway malformations as well as infective and inflammatory disorders are 60 times more common than neoplastic tumours. ${ }^{[2]}$

A tumour of the chest is considerably difficult to diagnose since patients can be asymptomatic for many years before symptoms evolve. Even more so, the symptoms are nonspecific and can suggest more common and less sinister pathology.

Clinically patients present with a variety of symptoms that depend largely on the location of the tumour. Airway tumours can be symptomatic or can present with chronic cough, wheeze, haemoptysis, atelectasis or persistent pneumonia. Secondary malignant parenchymal tumours are likely to be symptomatic from the primary lesion. Anterior mediastinal tumours can cause compression of the large airways or superior vena caval structures. It stands to reason that the physician needs to have a very high index of suspicion when dealing with these nonspecific signs and symptoms.

This article provides an approach to tumours of the chest and reviews the common aetiology in the different compartments of the chest. The article will focus on common tumours of the airway, lung parenchyma, mediastinum, cardiac and chest wall pathology (Table 1).

\section{Tumours of the airways}

Benign tumours of the airways

Subglottic haemangioma

Haemangiomas of the airway are most commonly seen in the subglottic or upper tracheal region. They are a rare finding in the distal trachea or bronchial region. These patients classically present with features of upper airway obstruction at 3 - 6 months of age. The upper airway obstruction and accompanied respiratory distress can be severe enough to cause

\begin{tabular}{|c|c|c|c|}
\hline Location & Benign & \multicolumn{2}{|l|}{ Malignant } \\
\hline \multirow[t]{2}{*}{ Airway } & Subglottic haemangioma & \multicolumn{2}{|c|}{ Bronchial adenoma } \\
\hline & Papilloma of the trachea and bronchi & \multicolumn{2}{|c|}{ Mucoepidermoid carcinoma } \\
\hline \multirow[t]{3}{*}{ Lung parenchymal } & Inflammatory pseudotumour & Primary & Pleuropulmonary blastoma \\
\hline & Hamartoma & & Kaposi's Sarcoma \\
\hline & Teratoma & Secondary & $\begin{array}{l}\text { Secondary metastases originating from Wilms' tumour, } \\
\text { osteosarcoma, rhabdomysocarcoma, neuroblastoma and } \\
\text { hepatoblastoma }\end{array}$ \\
\hline \multirow[t]{4}{*}{ Mediastinal } & Teratoma & \multicolumn{2}{|c|}{ Hodgkin's lymphoma } \\
\hline & Ectopic/hyperplastic thymus & \multicolumn{2}{|c|}{ Non-Hodgkin's lymphoma } \\
\hline & Vascular malformations & \multirow{2}{*}{\multicolumn{2}{|c|}{ Neuroblastoma }} \\
\hline & Bronchogenic cyst & & \\
\hline \multirow[t]{2}{*}{ Cardiac } & Rhabdomyoma & & \\
\hline & Myxoma & & \\
\hline \multirow[t]{3}{*}{ Chest wall } & Haemangioma & \multicolumn{2}{|c|}{ Rhabdomyosarcoma } \\
\hline & Osteoid osteoma & \multicolumn{2}{|c|}{ Osteosarcoma } \\
\hline & Osteochondroma & & \\
\hline
\end{tabular}


respiratory failure. The time of presentation is very important as this is the period in which these lesions undergo vascular proliferation and enlarge considerably. ${ }^{[3]}$ Spontaneous resolution is said to occur at about 12 months of age. ${ }^{[4]}$

The diagnosis should be considered in any patient in this age group who presents with hoarseness of voice, recurrent croup and a barking cough. ${ }^{[5]}$ The stridor is typically biphasic in nature. Upper endoscopy reveals asymmetric swelling most commonly in the subglottic region. Up to $20 \%$ of these patients may have cutaneous lesions, especially on the face in a beard distribution. ${ }^{[5]}$ Biopsy of these lesions is not recommended due to the very likely complication of bleeding. ${ }^{[4,5]}$

The first-line treatment of these lesions is that of beta-blockers, which may have to be continued up to 12 months of age. ${ }^{[4,5]}$ Other modalities of treatment include systemic and intra-lesion steroids and laser ablation. ${ }^{[4]}$

\section{Papilloma of the trachea and bronchi}

Respiratory papillomatosis is caused by human papillomavirus (most importantly types 6 and 11) and it is typically acquired during delivery. ${ }^{[1,6]}$ It most commonly affects the larynx and trachea but can progress to involve the bronchi as well as the oesophagus. ${ }^{[2]}$ These are the most common benign tumours of the larynx affecting children in a bimodal distribution, with $25 \%$ of patients younger than 5 years and the second peak occurring in the second and third decade of life. ${ }^{[6]} \mathrm{An}$ example of papillomas of the airway is shown in Fig. 1.

Classically these patients present with features of upper airway obstruction and a triad of hoarseness of voice, stridor and respiratory distress. ${ }^{[6]}$ This condition should be remembered in the differential diagnosis

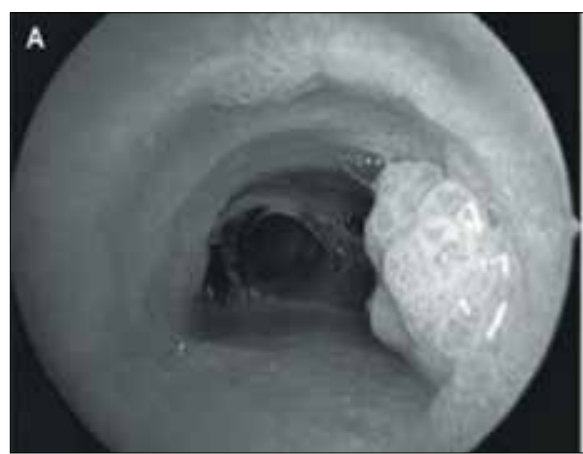

Fig. 1. Papilloma of the airway on bronchoscopy (A). Subsequent cavitatory lesions in the lung noted on axial CT of the chest likely from seeding of the tumour (B) (arrow). (Courtesy of Prof. Refiloe Masekela, Department of Paediatrics and Child Health, University of KwaZulu-Natal).

of patients who present with croup that is non-responsive to adrenaline nebulisation.

The most important complication of laryngeal papillomatosis is airway compromise. Certainly patients can also develop failure to thrive and recurrent pneumonia. Malignant transformation can occur although it is very rare. ${ }^{[6]}$ Concomitant pulmonary parenchymal lesions are present in less than $1 \%$ of patients with airway lesions. ${ }^{[1]}$

Management involves surgical ablation, $\mathrm{CO}_{2}$ and laser vaporisation; medical adjuvant therapy for recurrent and large lesions includes alpha interferon and cidofivir. ${ }^{[1,6]}$ Current therapies are unfortunately not curative and periodic resections may be necessary. ${ }^{[1]}$ Preventive strategies in the form of the quadrivalent vaccine against human papillomavirus hold promise to decrease the incidence of juvenile respiratory papillomatosis. ${ }^{[6]}$

\section{Malignant tumours of the airways \\ Bronchial adenoma}

Together with pleuropulmonary blastomas, bronchial adenomas are the most common primary malignancies of the lung in childhood. Two histological types of bronchial adenomas are defined: the carcinoid type, which occurs more commonly (90\%), and the cylindromatous type. ${ }^{[7]}$ Adenomas are more common in males and usually occur in older children and adolescents, with a mean age of presentation of 12 years. ${ }^{[1,2]}$ Carcinoid tumours are low-grade tumours with low potential to metastasise; they are however locally aggressive. ${ }^{[1,2]}$

This should form part of the differential diagnosis of patients who present with poorly controlled asthma, recurrent pneumonia and even haemoptysis (although rare). The diagnosis is based on histology. Some authors

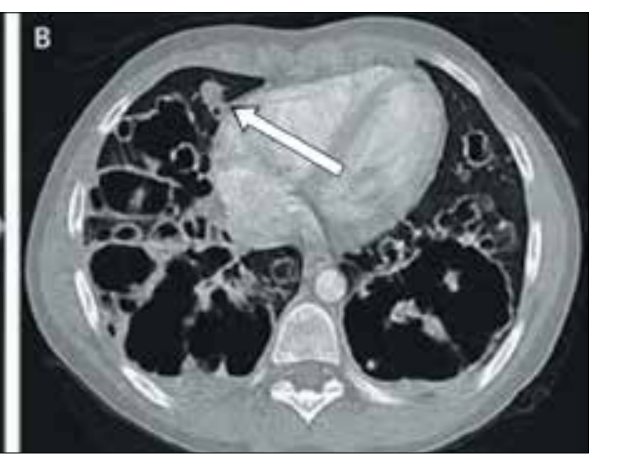

\section{the}

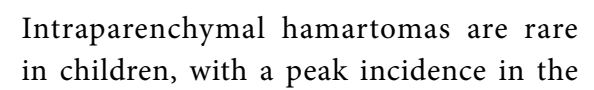

recommend biopsy via rigid bronchoscopy, as flexible bronchoscopy-directed biopsy carries a significant risk of bleeding. ${ }^{[2]}$

Management of these lesions involves resection of a segment, lobe or entire lung depending on the position of the tumour and extent of the mass. Bronchoscopy removal is not advised due to the risk of bleeding and incomplete removal of the lesion. ${ }^{[2,7]}$ The overall prognosis is excellent, with a survival rate of more than $90 \% .^{[1,2]}$

\section{Mucoepidermoid carcinoma} carcinoid tumours. They typically present just like bronchial adenomas with recurrent pneumonia, endobronchial obstruction with wheeze and haemoptysis. They most commonly occur as a polypoid exophytic causing obstruction of the main stem bronchus or proximal lobar bronchus. ${ }^{[2]}$ The prognosis in children is excellent, with an $88 \%$ survival rate. The treatment consists of surgical removal with adjuvant chemotherapy reserved for those with incomplete resection. ${ }^{[2]}$

\section{Lung parenchymal tumours}

Benign lung parenchymal tumours Inflammatory pseudotumour (inflammatory myofibroblastic tumour)

This is the most commonly occurring primary benign lung parenchymal tumour in the paediatric population. ${ }^{[1,2]} \mathrm{Up}$ to $30 \%$ of lesions are asymptomatic, but the rest present with nonspecific features of cough and fever. Patients are usually above the age of 5 years with an equal sex distribution. ${ }^{[1,2,7]}$ Local invasion and recurrence are rarely seen in children diagnosed with these lesions. ${ }^{[1]}$ Although classically a parenchymal lesion, in $12 \%$ of cases these lesions can occur as endobronchial lesions. ${ }^{[1]}$

The chest X-ray (CXR) typically reveals a well-circumscribed solitary nodule that is peripherally based, usually located in the lower lobes. ${ }^{[1,2]}$ It ranges in size from 1 to $12 \mathrm{~cm}$. The diagnosis is made histologically upon surgical removal. Biopsy specimens of the lesion are sometimes inaccurate and thus complete excision is required for the treatment and confirmation of the diagnosis. ${ }^{[1]}$

\section{amartoma}

These tumours are much rarer than 
fourth and sixth decades, but can also occur in the paediatric population. ${ }^{[1,2]}$ They are well-encapsulated solid masses that more commonly occur as intraparenchymal lesions. However, they may also occur in endobronchial sites. Most of these lesions are incidentally found on imaging studies as these patients are usually asymptomatic. ${ }^{[1]}$ The characteristic computed tomography (CT) finding of these lesions is as 'popcorn calcifications. ${ }^{[2,7]}$ Percutaneous needle aspiration and core needle biopsies are adequate to attain a histological diagnosis. ${ }^{[1]}$ Surgical resection may play a role in cases with an uncertain diagnosis. ${ }^{[1]}$

\section{Teratoma}

Teratomas are tumours that originate from more than one germ cell line. Intrathoracic teratomas are the fourth most common site, the most common being the gonads. Intrathoracically, teratomas usually occur in the anterior mediastinal region. ${ }^{[8]}$ Intraparenchymal teratomas are extremely rare. ${ }^{[8-10]}$ These tumours are more common in the third and fourth decades of life.

Patients can be completely asymptomatic or present with nonspecific symptoms of chest pain, cough and even haemoptysis. ${ }^{[8]}$ The CXR will depict a well-demarcated lesion as in Fig. 2. The CT scan of the chest will demonstrate a well-circumscribed cystic lesion (Fig. 3), the major differential of which is a hydatid cyst.

The management rests on surgical excision of these lesions. Adjuvant chemotherapy should be employed for immature teratomas. ${ }^{[1]}$ The definitive diagnosis rests on histology after the lesion is removed, demonstrating features from multiple germ cell lines as shown in Fig. 4.

Primary malignant lung parenchymal tumours

Pleuropulmonary blastoma

Pleuropulmonary blastoma ( $\mathrm{PPB})$ is an extremely rare malignant primary tumour of the pulmonary or pleural mesenchyme. Interestingly, $\mathrm{PPB}$ is quoted to account for $15 \%$ of primary paediatric pulmonary tumours. However, it is the most common primary parenchymal lung malignancy. The age of presentation is usually $<4$ years of age. There are essentially three types, and a fourth type (type Ir) was added in 2006. There is no gender predilection and tumours of the right side are more common. ${ }^{[12]}$
Type 1 PPB is predominantly cystic as in the case described above (Figs 5-8), type 2 mixed cystic and solid lesions and type 3 predominantly solid lesions. There is an increasing risk of malignant potential from types 1 to $3 .^{[12]}$ Type $1 \mathrm{r}$ lesions (type 1 regressed) are cystic in nature and may represent a regressed type of type 1 tumour. ${ }^{[12]}$ PPB type 1 lesions carry the most favourable prognosis with a long-term survival of $80 \%$. Unfortunately, types 2 and 3 lesions are more common, are highly aggressive and

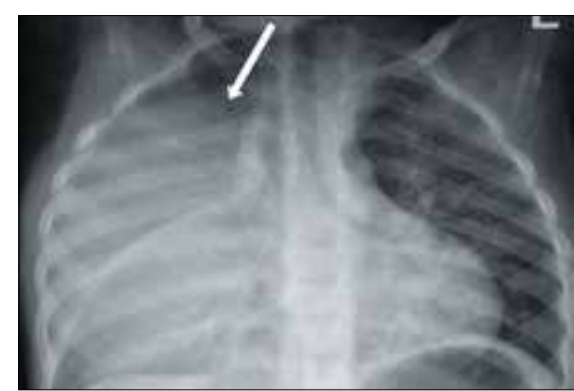

Fig. 2. Frontal CXR of 3-year-old female with right-sided mass occupying most of the right hemithorax (arrow).

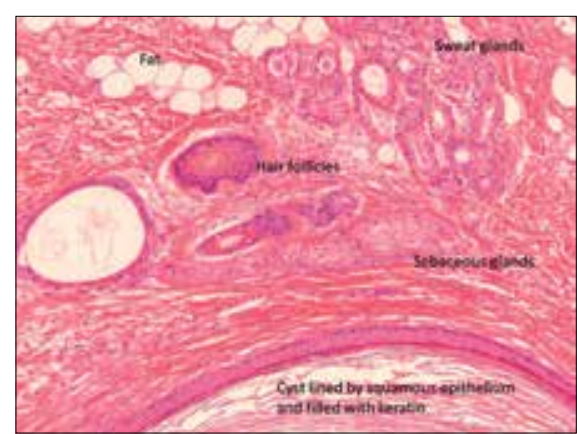

Fig. 4. HeE section of the lesion in Figs 2 and 3 demonstrating cells from multiple germ cell lines. This is compatible with a teratoma. (Courtesy of Dr A van Rooyen, Department of Anatomical Pathology, Tshwane Academic Division, National Health Laboratory Service (NHLS)).

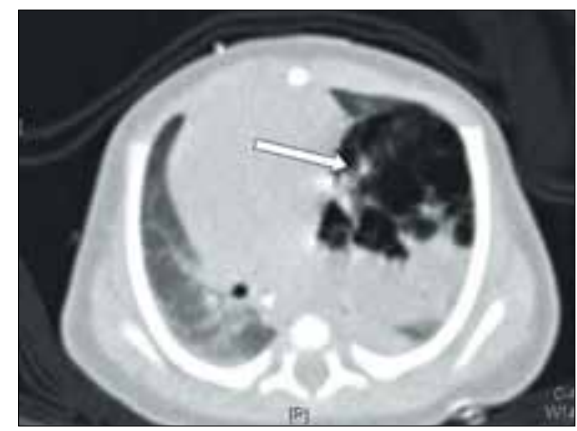

Fig. 6. Axial CT chest. Large (>2 cm) multicystic lesion. The mediastinal shift is apparent on CT scan of the chest. The cystic lesions appear fluid filled and thus complicated. carry a poorer prognosis (long-term survival of $<50 \%)$. It is thought that type 1 lesions progress to types 2 and 3 with time. ${ }^{[13]}$ The most common site of metastases is the brain followed by bone. ${ }^{[12]}$ Radiologically, PPB can occur as a single cystic, a multicystic structure, a cyst with a polypoid mass, a mixed solid-cystic lesion or a completely solid lesion. ${ }^{[12]}$ Unfortunately fine needle aspiration and cytology is inadequate to make a histological diagnosis and the entire lesion needs to be removed for the diagnosis. ${ }^{[14]}$

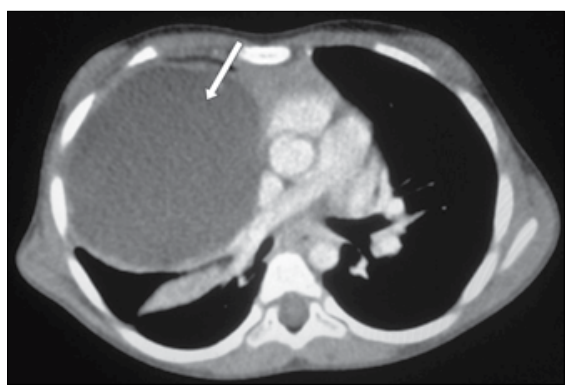

Fig. 3. Contrasted axial CT of the chest of the same patient in Fig. 2. The lesion is a thinwalled fluid-filled cystic mass occupying a large portion of the right hemithorax (arrow).

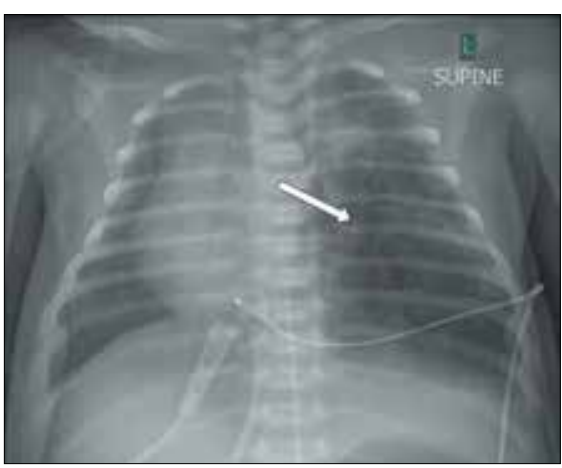

Fig. 5. Frontal CXR. Left lower lobe multicystic lesion (arrow). The cysts appear large. There is significant mediastinal shift to the right.

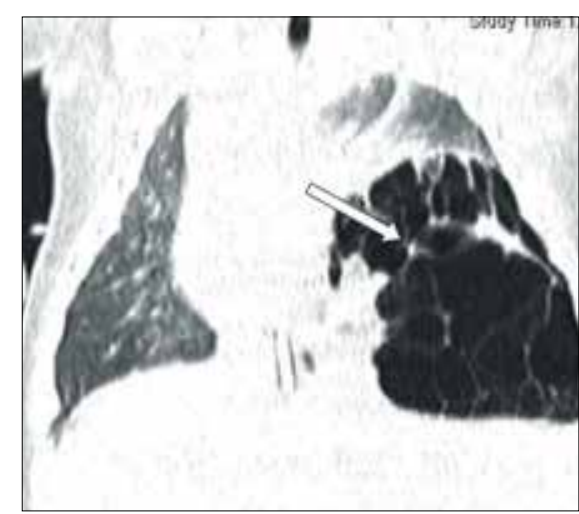

Fig. 7. Coronal CT of the chest showing extent of the lesion. 


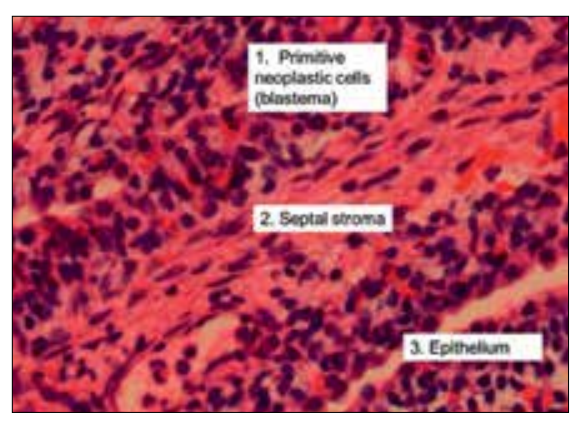

Fig. 8. HÆE section demonstrating the primitive neoplastic cells characteristic of a pleuropulmonary blastoma. (Courtesy of $\operatorname{Dr} C$ Crause, Department of Anatomical Pathology, Tshwane Academic Division, NHLS).

The management of these patients includes surgical removal of the lesion in the form of a lobectomy and a combination of chemotherapy and radiotherapy. ${ }^{[12,13]}$ The chemotherapeutic regimen suggested includes a combination of ifosfamide, vincristine, actinomycin D and doxorubicin. ${ }^{[12]}$

There is much controversy in paediatric pulmonology about the indications to remove congenital pulmonary airway malformations (CPAMs). ${ }^{[15]}$ Certainly in the case of symptomatic (causing compression with respiratory distress), infected CPAMs or hybrid lesions the consensus lies in removal of these lesions. ${ }^{[16,17]} \mathrm{PPB}$ can appear identical to CPAMs and the diagnosis rests solely on histology with entire removal of the lesion. More so, PPBs are highly aggressive tumours and the outcome is generally poor if not attended to. ${ }^{[16]}$ The dilemma thus arises in the face of asymptomatic lesions that appear as CPAMs. The diagnosis of a PPB runs the risk of being missed if these lesions are not removed. It is important for the physician to note that even though primary CPAMs may be removed, it does not protect against the chance of malignant transformation. ${ }^{[18]}$ More so, the risk of malignant transformation in a CPAM is largely unknown and has never been quantified. ${ }^{[17]}$ The issue then to remove a lesion should be discussed with the parents of the child, the individual risks including short-term surgical risks and long-term risks of infection and malignant transformation explained to them and a decision made as a team.

\section{Kaposi's sarcoma}

Kaposi sarcoma (KS) is a low-grade vascular tumour associated with human herpes virus 8 . The physician should certainly be aware of KS of the lung, especially in the South African setting. Childhood KS is now quoted as the most common AIDS-defining malignancy affecting children in sub-Saharan Africa, with pulmonary KS thought to occur in $10 \%$ of these cases. ${ }^{[19]}$ Cutaneous KS is the most common site. ${ }^{[20]}$

The presenting features of pulmonary KS rests on a common triad of cough, dyspnoea and lymphadenopathy. ${ }^{[2]}$ Patients can also present with features of upper and lower airway obstruction; haemoptysis in children is rare. Extensive pulmonary KS can precede the development of mucocutaneous lesions. The typical radiological features include air-space disease, especially in the mid and lower zones, hilar lymphadenopathy and significantly large pleural effusions that can be bilateral. ${ }^{[21]}$

The overall prognosis is unfortunately poor with chemotherapy the mainstay of treatment. ${ }^{[19]}$ Controlling the HIV disease is as important as chemotherapy for these patients and has a large impact on the survival rates. ${ }^{[22]}$

The diagnosis of pulmonary KS rests on a high index of suspicion with typical features described above. Histology of a peripheral site including skin lesions or lymph nodes is supportive of the diagnosis. ${ }^{[21]}$

\section{Other primary malignant parenchymal} tumours

Other primary malignant tumours of the lung including bronchogenic carcinoma, leiomyosarcoma, multiple myeloma and choriepithelioma are extremely rare in the paediatric population. In terms of bronchogenic carcinoma, adenocarcinoma accounts for more cases than squamous cell carcinoma. These tumours as a group are extremely aggressive and carry an overall poor prognosis. ${ }^{[2]}$

Secondary malignant lung parenchymal tumours

Secondary malignant parenchymal tumours are much more common than primary parenchymal lung malignancies. In one study, secondary malignancies were 12 times more prevalent than primary parenchymal malignancies. Secondary metastases that occur in the lung in the paediatric population differ from those associated with adult disease. ${ }^{[2]}$ In a 25-year cohort of pathology specimens in Texas Children's Hospital the following secondary malignancies were noted to be common in decreasing order of incidence: Wilms' tumour, osteosarcoma, rhabdomyosarcoma, neuroblastoma and hepatoblastoma. ${ }^{[2]}$
The general principles of management of secondary lesions include control of the primary site. Secondary metastases should only be considered for removal once other sites are disease free. However, soft-tissue sarcomas and osteosarcomas should be considered for removal of pulmonary disease as removal of these lesions is associated with a better prognosis. ${ }^{[7]}$

\section{Mediastinal tumours}

The mediastinum is that area that extends from the thoracic inlet superiorly to the diaphragm inferiorly; it is bound laterally by the parietal pleura, anteriorly by the sternum and posteriorly by the vertebral column. The superior mediastinum extends from the thoracic inlet to an imaginary line from the angle of the sternum to the T4/T5 intervertebral space. It is further divided into anterior, middle and posterior compartments. It is paramount that the physician is aware of these anatomical boundaries so as to limit the differential diagnosis based on the anatomical compartment involved. ${ }^{[23]}$

Common pathological causes include: T-cell lymphoma, Hodgkin's lymphoma, germ cell

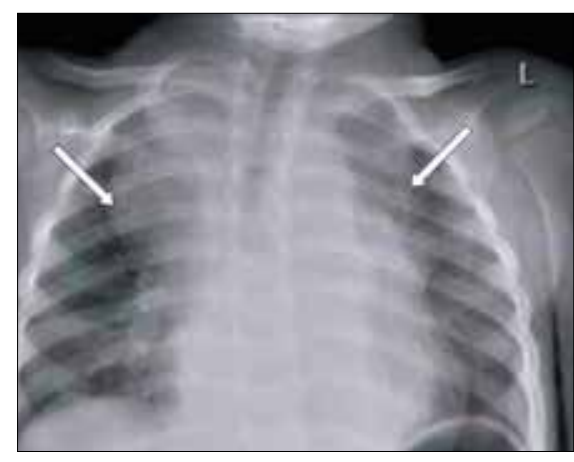

Fig. 9 Frontal CXR of a 3-year-old boy demonstrating an enlarged superior mediastinum (arrows).

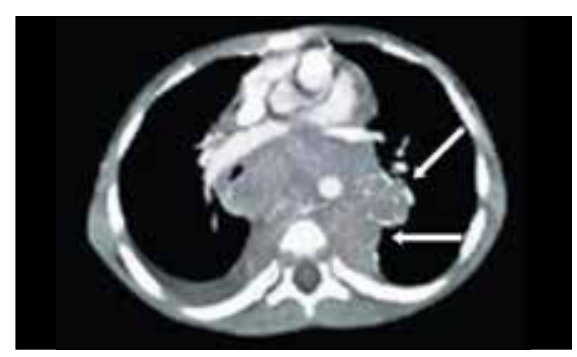

Fig. 10. Contrasted axial CT scan at the level of the great vessels of the same patient in Fig. 9, demonstrating lymphadenopathy in the posterior mediastinum. This was histologically confirmed to be a neuroblastoma. 
tumours and neuroblastoma and occur more commonly in different compartments of the superior mediastinum. For a more in-depth approach to masses that occur in the superior mediastinum, the reader is referred to the case report of a superior mediastinal mass elsewhere in this journal. Figs 9 and 10 show an example of a widened superior mediastinal secondary to a neuroblastoma in the posterior mediastinum.

\section{Cardiac tumours}

Cardiac tumours should be considered in the differential diagnosis of mediastinal masses. Cardiac tumours in the paediatric population are very rare and most lesions are benign. These tumours are usually associated with a systemic disease process such as tuberous sclerosis. In terms of intracardiac masses the most common causes are rhabdomyoma, myxoma and fibroma. Pericardial lesions include pericardial cysts and teratomas. ${ }^{[2]}$

\section{Chest wall tumours}

Chest wall tumours in children can also be benign or malignant.

The most common benign lesions include infantile haemangioma, osteoid osteoma and osteochondroma.

Infantile haemangiomas are vascular tumours that proliferate rapidly in the first few months of life and thereafter undergo spontaneous regression. They are well demarcated, palpable bluish-red lesions. Since they spontaneously regress, there is often no need for treatment.

Osteoid osteomas usually occur in the long bones but can occur in the thorax. They are more common in males between the ages of 7 and 25 years. These are self-limiting lesions and usually require no further treatment, other than analgesia.

Osteochondromas also have a male predominance, beginning to grow just before puberty and occurring most commonly at the chostochondral junctions. These lesions can cause significant pain, can fracture and have the potential for malignant transformation, especially if the lesions are multiple.

Primary malignant tumours that affect the chest wall include rhabdomyosarcoma and osteosarcoma.

Rhabdomyosarcomas are high-grade tumours that arise from skeletal muscle. Chest wall involvement can occur. However, it more commonly occurs in the head and neck region or in the abdominal wall. They are highly aggressive and have the potential to invade the surrounding bone, indicating advanced disease. Treatment of these lesions includes both surgical resection and chemotherapy.

Osteosarcomas are also highly aggressive tumours. They can originate anywhere from the bone, soft tissue or pleura. The peak incidence is during adolescence to young adulthood. The chief presenting feature is pain. Most commonly these lesions affect the long bones and $1-2 \%$ of osteosarcomas affect the chest wall. As with rhabdomyosarcomas, treatment involves surgery and chemotherapy. ${ }^{[25]}$

\section{Conclusion}

Tumours of the chest in children are very rare as a whole. The clinical features range from asymptomatic lesions to nonspecific symptoms of cough. The physician needs to always have a high index of suspicion. Compartmentalising the different possible causes into the most likely source of origin, whether it be airway or parenchymal, definitely aids in a reasonable differential diagnosis.

\section{References}

1. Amini B, Huang SY, Tsai J, Benveniste MF, Robledo HH, Lee EY. Primary lung and large airway neoplasms in children: Current imaging evaluation with multidetector computed tomography. Radiol Clin North Am 2013;51(4):637-657. [http://dx.doi. org/10.1016/j.rcl.2013.04.005]

2. Dichop MK, Kuruvilla S. Primary and metastatic lung tumours in the paediatric population. Arch Pathol Lab Med 2008;132(7):1079-1103.

3. Eyssartier E, Ang P, Bonnemaison E, et al. Characteristics of endobronchial primitive tumours in children. Paediatr Pulmonol 2014;49(6):E121-125. [http://dx.doi. org/10.1002/ppul.22987]

4. Hardison SA, Dodson KM, Rhodes JL. Subglottic hemangioma treated with propranolol. Eplasty 2014;14:ic2.

5. Tabatabaii SA, Khanbabaii G, Khatami AR, Sharifnia SA. Characteristic and follow-up of subglottic haemangiomas in Iranian children. J Res Med Sci 2010;15(4):235-239.

6. Masekela R, Smit A, Tshifularo MI, Green R. Juvenile recurrent respiratory papillomatosis a cause of airway obstruction: A case study. S Afr Ped Rev 2008;5(2):30-35.

7. Plerhoples TA, Krummel TM. Tumours of the chest. In: Wilmott RW, Boat TF, Bush A. Kendig and Chernick's Disorders of the Respiratory Tract in Children. 8th ed. Philadelphia: Elsevier Saunders, 2012:605-630.

8. Saini ML, Krishnamurthy S, Kumar RV. Intrapulmonary mature teratoma. Diag Path 2006;1(38):1-3. [http://dx.doi.org/10.1186/1746-1596-1-38]

9. Dar RA, Mushtaque M, Wani SH, Malik RA. Giant intrapulmonary teratoma: A rare case. Case Rep Pulmonol 2011;201: 298653. [http://dx.doi.org/10.1155/2011/298653]

10. Sawant AC, Kandra A, Narra SR. Intrapulmonary cystic teratoma mimicking malignant pulmonary neoplasm. BMJ Case Rep 2012;2012:bcr0220125770. [http:// doi.org/10.1136/bcr.02.2012.5770]

11. Terenziani M, D’Angelo P, Inserra A, et al. Mature and immature teratoma: A report from the second Italian pediatric study. Paediatr Blood Cancer 2015;62(7):1202-1208. [http://dx.doi.org/10.1002/pbc.25423]

12. Khan AA, El-Borai AK, Alnoajii M. Pleuropulmonary blastoma: A case report and review of the literature. Case Rep Path 2014;2014:509086. [http://dx.doi.org/10.1155/2014/509086]

13. Libretti L, Ciriaco P, Casiraghi M, Arrigoni G, Zannini P. Pleuropulmonary blastoma in the area of a diagnosed congenital lung cyst. Ann Thorac Surg 2008;85(2):658-660. [http://dx.doi.org/10.1016/j.athoracsur.2007.08.012]

14. Schultz KAP, Williams GM, Stewart DR, et al. Recurrent or progressive Type II and Type III pleuropulmonary blastoma (PPB) are associated with poor outcome: A report from the International PPB Registry. J Clin Oncol 2015;33(15S):10014.

15. Fauroux B. Congenital cystic adenomatous malformation (CCAM): Remove or not, how can we progress? Paediatr Respir Rev 2013;14(3):168. [http://dx.doi. org/10.1016/j.prrv.2013.06.001]

16. Delacourt C, Hadchouel A, Khen Dunlop N. Shall all congenital cystic malformations be removed? The case in favour. Paediatr Respir Rev 2013;14(3):169-170. [http:// dx.doi.org/10.1016/j.prrv.2013.06.003]

17. Kotecha S. Should asymptomatic congenital cystic malformations be removed? The case against. Paediatr Respir Rev 2013;14(3):171-172. [http://dx.doi.org/10.1016/j. prrv.2013.06.002]

18. Papagiannopoulos KA, Sheppard M, Bush AP, Goldstraw P. Pleuropulmonary blastoma: Is prophylactic resection of congenital lung cysts effective? Ann Thorac Surg 2001;72(2):604-605.

19. Graham SM. Non-tuberculosis opportunistic infections and other lung diseases in HIV-infected infants and children. Int J Tuberc Lung Dis 2005;9(6):592-602.

20. De Bruin GP, Stefan DC. Children with Kaposi sarcoma in two South African hospitals: Clinical presentation, management and outcome. J Trop Med 2013;2013:213490. [http://dx.doi.org/10.1155/2013/213490]

21. Theron S, Andronikou S, Du Plessis J, et al. Pulmonary Kaposi sarcoma in six children. Paediatr Radiol 2007;37(12):1224-1229. [http://dx.doi.org/10.1007/s00247-007-0632-9]

22. Stefan DC, Stones DK, Wainwright L, Newton R. Kaposi sarcoma in South African children. Paediatr Blood Cancer 2011;56(3):392-396. [http://dx.doi.org/10.1002/pbc.22903]

23. Williams HJ, Alton HM. Imaging of paediatric mediastinal abnormalities. Paediatr Respir Rev 2003;4(1):55-66. [http://dx.doi.org/10.1016/S1526-0542(02)00310-X]

24. Newman B. Thoracic neoplasms in children. Radiol Clin North Am 2011;49(4):633664. [http://dx.doi.org/10.1016/j.rcl.2011.05.010]

25. Baez JC, Lee EY, Restrepo R, Eisenber RL. Chest wall lesions in children. AJR Am J Roentgenol 2013;200(5):W402-W419. [http://dx.doi.org/10.2214/AJR.12.8883] 